75\% (364/486); surgeons 74\% (147/199); medical 70\% (368/527); $\mathrm{p}<0.1$. Size assessment correlated poorly with histology with a significant increase in the use of $5 \mathrm{~mm}$ and $10 \mathrm{~mm}$ measurements in vivo $\left(\chi^{2}=71.3 \mathrm{DF}=9 \mathrm{p}<0.0001\right) .290$ polyps were estimated smaller in vivo and 302 larger (294 precise) when compared to histology [distribution of error curve: $\mathrm{SD}=3.22 \mathrm{~mm}$; mean $=0.16 \mathrm{~mm}$; median=0]. A discrepancy across the $10 \mathrm{~mm}$ size occurred in 96 polyps (11\%).

Conclusion Currently a poor PPV for neoplastic polyps and imprecise in vivo size estimation would mean a resect and discard approach would be inaccurate, but current practice removes a large amount of benign pathology and has both patient morbidity and significant cost implications.

Competing interests None declared.

\section{PTU-234 ENDOSCOPIC ULTRASOUND GUIDED FINE NEEDLE ASPIRATION FOR THE DIAGNOSIS OF PANCREATIC CYSTIC NEOPLASMS: A META-ANALYSIS}

doi:10.1136/gutjnl-2012-302514c.234

${ }^{1} \mathrm{G} D$ Thornton, ${ }^{1} \mathrm{M} \mathrm{J}$ McPhail, ${ }^{2} \mathrm{~S}$ Nayagam, ${ }^{1} \mathrm{M} \mathrm{J}$ Hewitt, ${ }^{*}{ }^{1} \mathrm{P}$ Vlavianos,

${ }^{1,2} \mathrm{~K}$ J Monahan. ${ }^{1}$ Imperial College, London, UK; ${ }^{2}$ West Middlesex Hospital, London, UK

Introduction Pancreatic cystic neoplasms consist of mucinous cystic neoplasms (MCNs) and serous cystic neoplasms (SCNs). MCNs have significantly greater malignant potential, and if resected early the prognosis is excellent, although mortality is $2 \%-3 \%$. Endoscopic ultrasound is a minimally invasive and well tolerated procedure. EUS with fine-needle aspiration (EUS-FNA) provides samples for cytology and fluid analysis, a major advantage over other techniques. However the diagnostic accuracy of EUS-FNA is highly variable in published studies.

Aim To determine the diagnostic accuracy of EUS-FNA to differentiate mucinous vs non-mucinous cystic lesions with morphology, and cyst fluid analysis for cytology and carcinoembryonic antigen (CEA) via a meta-analysis of published studies.

Methods Relevant studies were identified using MEDLINE and included if they used a reference standard of definitive surgical pathology or clinical follow-up ( $\geq 6$ months). Study quality was assessed using the STARD (STAndards for the Reporting of Diagnostic Accuracy) initiative criteria. Data were analysed using MetaDiSc(C) v.1.4, which generated pooled estimates for sensitivity, specificity and summary ROC curve. Subgroups, determined a priori, were used to assess heterogeneity: prospective vs retrospective, location, number of centres and patients, 19G or 22G needle and STARD score.

Results 24 studies published between 2001 and 2011 were included, a total of 1703 patients. The median number of patients in each study was 53 (range 18-197) and the median study length was 54 $(12-144)$ months. The pooled sensitivities $(95 \% \mathrm{CI})$ and specificities $(95 \% \mathrm{CI})$ and area under the sROC curve (SE), respectively, were: EUS morphology 55 (49-61)\%, $65(57-72) \%$ and 0.74 (0.095); Cytology $54(50-59) \%, 93(90-95) \%$ and 0.95 (0.040); and CEA 63 $(59-67) \%, 88(83-91) \%$ and 0.79 (0.034). Subgroup analysis indicated that retrospective design, low STARD score and study location outside Europe were significant sources of heterogeneity.

Conclusion Fine-needle aspiration has moderate sensitivity but high specificity resulting in good overall diagnostic accuracy for MCNs. Morphology alone is inadequate for distinguishing cystic lesions but may contribute to the assessment of more advanced lesions. The moderate sensitivity of FNA (54\%) means a significant proportion of MCNs will not be detected. However, the high specificity (93\%) means that a positive result is strongly indicative of a MCN. Thus, EUS-FNA is a useful diagnostic tool for correct identification of MCNs and may be the gold standard for pre-operative assessment.

Competing interests None declared.
SUCCESS OF SINGLE STENT ENDOSCOPIC ULTRASOUND (EUS) GUIDED PANCREATIC CYSTOGASTROSTOMY

doi:10.1136/gutjnl-2012-302514c.235

M Roberts, ${ }^{*}$ V Kaushik, Y Reddy. Royal Blackburn Hospital, Blackburn, UK

Introduction Current accepted practice for EUS-guided stent insertion for drainage of pancreatic pseudocysts (PP) involves placement of two or more double-pigtail stents. ${ }^{1}$ There has been little work into whether a single stent would provide an equal outcome without increasing complications. Rates of successful drainage without surgical or percutaneous intervention are $88-64 \%$ with complication rates at $32-14 \% .^{2}$ We present a case series comparing outcomes of single stent insertion against these.

Methods Retrospective analysis of cases, identified by searching the electronic endoscopic database, in a DGH providing tertiary HPB and EUS service between August 2005 and December 2011 was performed. All procedures were performed by the same experienced endoscopist. All patients had a radiographically proven PP and received a single $7 \mathrm{~cm}$ 7Fr double-pigtail stent with prophylactic antibiotics.

Results 24 patients underwent cystogastrostomy. 1 patient died from unrelated causes in the days post-procedure, therefore is not included in the analysis. 20 of 23 patients (86.9\%) were successfully drained without any surgical or percutaneous intervention. One patient had a pre-existing infected $\mathrm{PP}$, only recognised on fluid analysis after the procedure, required laparotomy for excision and surgical cystogastrostomy. With hindsight more than one stent may have resulted in a better outcome. There were two procedure related complications (8.6\%). One suffered pneumoperitoneum 2 days postprocedure. Emergency surgery revealed the pancreatic lesion to actually be a mucinous cystadenoma, despite radiographically and endoscopically (including pre-stent insertion aspirate) behaving as a PP. This misdiagnosis and complication was unavoidable due to this unusual behaviour. The other patient developed sepsis 26 days postprocedure, and had a CT guided drain. Culture of aspirated cyst fluid revealed candida, possibly a post-insertion complication or an incidental finding; patient responded to fluconozole. Two further patients were re-admitted with pyrexia 3 and 5 days respectively post-procedure. They improved with antibiotics, required no intervention, and imaging revealed PP improvement.

Conclusion This data suggests that efficacy and complication rates for single-stented EUS-guided cystogastrostomy in simple PP are comparable with data from studies using two or more stents. This would result in shorter procedure time and reduced risks from insertion thereby improving patient safety, and reduced costs. Single stenting is not recommended for infected or complex PP.

Competing interests None declared.

\section{REFERENCES}

1. Seewald $\mathbf{S}$, Ang TL, Teng KC, et al. EUS-guided drainage of pancreatic pseudocysts, abscesses and infected necrosis. Dig Endosc 2009;21(Suppl 1):S61-5.

2. National Institute of Clinical Excellence. Endoscopic Trans/uminal Drainage of Pancreatic Pseudocyst and Pancreatic Necrosectomy-Overview. 2011.

\section{PTU-236 ENDOSCOPIC RETROGRADE CHOLANGIOPANCREATOGRAPHY (ERCP) METAL BILIARY STENT INSERTIONS: OUTCOME AND COMPLICATIONS}

doi:10.1136/gutjnl-2012-302514c.236

M Z Cader, ${ }^{*}$ M W James. Nottingham Digestive Diseases Centre Biomedical Research Unit, University of Nottingham, Nottingham, UK

Introduction Endoscopic retrograde cholangiopancreatography (ERCP) is an established technique for palliative stenting and 